\title{
Electrophotonic imaging based analysis of diabetes
}

\begin{abstract}
All metabolic processes involve electron transfer mechanisms. Eletrophotonic Imaging (EPI) captures the coronal discharge around the finger as a result of electron capture from the ten fingers. The coronal discharge around each fingertip supposedly represents the energy state of the corresponding organs and organ systems. The Periodic assessment of blood sugar is essential for the management of diabetes. An EPI based measurement process could be an alternative.

Electrophotonic Imaging (EPI) data is collected from 200 subjects including males and females in the age group of 40 to 60years from a diabetic center in Bangalore. The EPI data was captured from all the ten fingers from the subjects who came for the regular blood test. The EPI data corresponding to the meridians of the ring finger, chakras, organs and organ systems related to diabetes were analyzed using general linear model in IBM SPSS version 20.0. A built in neural network classifier from IBM SPSS was used to classify diabetic subjects from non-diabetic subjects.

The mean and standard deviation values for pancreas were 5.0240 and 1.02754 for the diabetic subjects and 4.7383 and 0.87618 for the non-diabetic subjects. Similarly for the hypothalamus the mean and standard deviation values were 4.9760 and 0.75926 for diabetes and 4.6150 and 0.86120 for non-diabetic subjects. The units for all the measurements are pixel units and represent empirical value for photonic energy. The classification accuracy of the neural network classifier was in the range of $80 \%$ to $100 \%$. EPI parameters had a statistically significant difference from the non-diabetic subjects. EPI data could be refined more to make use of it as a bio-metric for diabetes.
\end{abstract}

Keywords: diabetes assessment, electro photonic imaging, neural network, multi-layer, perception
Volume 4 Issue 5 - 2016

\author{
Shiva Kumar Kotikalapudi,' Srinivasan T,' \\ Nagendra HR,' Marimuthu P' \\ 'Department of Bioenergy, SVYASA Yoga University, India \\ ${ }^{2}$ Department of Biostatistics National Institute of Mental Health \\ and Neuro Sciences, (NIMHANS), India
}

Correspondence: Shiva Kumar Kotikalapudi, SVYASA Yoga University Bangalore, India, Tel +91-9945578216, Emailshivak210m@yahoo.com

Received: October 27,2016 | Published: December 09, 2016

\section{Introduction}

Diabetes is defined as hyperglycemia for a long term. Abnormal glucose metabolism leading to hyperglycemia defines diabetes. ${ }^{1}$ Diabetes Mellitus is a family of diseases with increased levels of blood sugar due to defects in insulin action or secretion associated with long term damage or failure of various organs like eyes kidneys, blood vessels and nerves. ${ }^{2}$ Presence of sugar in the blood for a long time leads to various disorders like thickening of the arterial walls causing blood pressure to increase and organs and organ systems becoming weaker and non-functional due to non-availability of the required quantity of glucose. ${ }^{3}$ Obesity, high fat diet and sedentary lifestyle contribute to the prevalence of type 2 diabetes. ${ }^{4}$ Diabetes is a lifestyle non-communicable disorder.

Brains role in glucose homeostasis was proposed by scientists in the nineteenth century however the current research diagnosis and treatment is based on the functioning of pancreatic islets. Recent works indicates that brain controlled glucose regulation can improve glucose homeostasis. ${ }^{5}$

Electrophotonic Imaging (EPI) instrument is used to capture coronal discharges at the fingertips induced by a pulsed electrical signal $(10-15 \mathrm{kV}, 1024 \mathrm{~Hz}, 10-$ microsecond) on the glass plate of the camera. This device produces a type of digital high-voltage electrophotography that is based on the Kirlian effect. ${ }^{6}$ The EPI is a non-invasive technique and is hazard free. ${ }^{7}$

The EPI instrument is based on the stimulation of photon and electron emissions from the surface of the object. This process is called 'photo-electron emissions' and it has been thoroughly studied with physical electronic methods. The emitted particles accelerate in the electromagnetic field, generating electronic avalanches on the surface of the dielectric (glass) plate. This process is called 'sliding gas discharge.' The discharge causes glow from the excitement of molecules in the surrounding gas, and this glow is what is being measured by the EPI instrument. Voltage pulses stimulate optoelectronic emission, while intensifying this emission in the gas discharge, amplified by the electric field created. ${ }^{8}$

Electrophotonic Image represents a spatially distributed glow areas having varying brightness characteristics, it reveals general, local and sector based details. ${ }^{9}$ One of the main questions is what is contained in the EPI data physical or psychical component. The researchers showed that it is the mental state the quality of psychic energy of man. ${ }^{10}$

It is important to diagnose this condition early to prevent further deterioration of the organs and organ systems. Electrophotonic Imaging (EPI) studies on Qigong suggest that EPI could be used for early diagnosis earlier than the conventional mechanisms of diagnosis. Diagnosing diabetes is not a onetime event, treatment of diabetes leads to processes like Self-monitoring of blood glucose. The process of frequent blood sampling is costly painful and leads to finger insensitivity. ${ }^{11}$

Analysis on degree of arterial hypertension concluded that EPI could be used to screen patients of hypertension with different levels of severity. ${ }^{12}$

Diagnosis of diabetes and its treatment need to be more holistic. The EPI instrument generates chakra based data which is more holistic. It also generates coronary discharges corresponding to the meridians as well as the organs and organ systems. The chakras can 
be thought of as energy centers, these energy centers are located on the spinal column starting from the base of the spine to the top of the head. ${ }^{13}$

The study on Autistic children concluded that EPI instrument is promising from a bio-metric perspective. ${ }^{14}$ The research on early prediction of diabetes using features of Electro Photonic Imaging also concluded that data can be used to train neural networks for classification of diseases for diagnosis. ${ }^{15}$ There have been some academic experiments in predicting the onset of diabetes using neural networks with a set of attributes as inputs to the neural network. ${ }^{4}$

Artificial Neural Network is a model consisting of a set of processing units which are closely interconnected to each other such that a rich structure is formed which exhibits certain features of biological neural network. The fundamental unit of a biological neural network is a neuron and each neuron gets connected to other neurons using dendrites and axons at the synaptic junctions or synapses. ${ }^{16}$

Similarly the fundamental unit of Artificial Neural Network is a neuron (processing unit) which gets connected to every other neuron in the network. The pattern recognition is the fundamental ability of the neural networks. It groups patterns, classifies patterns and maps patterns. Inputs to the neurons can be coming from either the external sources or from the other neurons in the network. The output of the neurons can be connected to other neurons in the network or will be available to the external world.

Learning is the basis for pattern recognition. There are several algorithms defined for learning the patterns and the networks are trained using the learning algorithms to learn the patterns. The input and expected output pairs are defined as patterns which are used for training the network. ${ }^{17}$ For example in case of diabetes classification input is the chakra data and the expected output is diabetic or nondiabetic.

During training phase the network is excited with input patterns repeatedly and the weights of all the neurons are adjusted every time in such a way that the network learns all the patterns. At the end of the training the patterns are stored in the form of weights in the network. ${ }^{18}$ The procedure to increment the weights of the neurons during the training phase is termed as learning and at the end of the successful training network can recall the pattern correctly from the weight vector.

Neural networks define several architectures for pattern recognition. Multilayer Perceptron ${ }^{19}$ and Radial basis function ${ }^{20}$ are two fundamental architectures which are popular in pattern recognition both these architectures are supported in the IBM SPSS tool version 20.0 under the Analysis menu.

There is a need for an automated study to observe the variations in all parameters with respect to a diseased condition to diagnose the same. ${ }^{21}$ The combination of EPI and neural network could be used as a framework for diagnosing diabetes.

This is the first study using EPI data and artificial neural network to classify diabetic and non-diabetic population. This study used three different sets of data. Data corresponding to the organs and organs systems, data for the chakras and data corresponding to the meridians.

\section{Materials and methods}

Electro photonic Imaging data was collected from a diabetic clinic Srinidhi Diabetic center at Bangalore, India from subjects who came for their regular blood sugar measurement. There were a total of 115 subjects diagnosed to be diabetic and 84 non-diabetic. The age of the subjects was between 20-60years. The EPI data was captured from all the ten fingers in sitting position. An informed consent was taken from all the subjects along with the permission from the Srinidhi diabetic center to participate in the study. The data was collected in the morning time from $9 \mathrm{Am}$ to $1 \mathrm{Am}$ from the entire subjects .EPI based instrument Bio-Well was used to collect the data.

Table I Coronal discharges corresponding to the Meridians of the Ring Fingers

\begin{tabular}{lll}
\hline Right ring finger & Name of organ/system & Value \\
\hline & Hypophysis & 60.19 \\
& Thyroid gland & 56.38 \\
& Pancreas & 56.6 \\
& Adrenal & 59.25 \\
& Urino-genital system & 62.24 \\
& Spleen & 60.2 \\
& Nervous system & 58.52 \\
& Hypothalamus & 59.75 \\
Left ring finger & \\
& Hypothalamus & 60.94 \\
& Nervous system & 58.45 \\
& Spleen & 59.43 \\
& Urino-genital system & 62.38 \\
& Adrenal & 62.6 \\
& Pancreas & 60.43 \\
& Thyroid gland & 59.13 \\
& Hypophysis & 59.86 \\
\hline
\end{tabular}

Table 2 EPI values corresponding to the size of The Chakras energy centers

\begin{tabular}{ll}
\hline Name of the energy centre & Value \\
\hline Muladhara (base of the Spine) & 5.1 I \\
Svadhisthana ( Below the Naval) & 4.58 \\
Manipura (Naval region) & 4.78 \\
Anahata ( Hear Region) & 5.46 \\
Vishuddha (Throat Region) & 4.82 \\
Ajna (In between the eyebrows) & 3.67 \\
Sahasrara (Top of the head) & 4.18 \\
\hline
\end{tabular}

Table 3 Organs and Organ Systems

\begin{tabular}{lll}
\hline Organ system & Organ & Energy \\
\hline Head & & 4.07 \\
& Eyes & 3.53 \\
Cardiovascular system & Cerebral zone (cortex) & 3.61 \\
& & 4.03 \\
& Cardiovascular system & 4.09 \\
& Heart & 4.06 \\
& Cerebral zone (vessels) & 3.49 \\
Endocrine system & Coronary vessels & 4.46 \\
& & 4.25 \\
& Hypothalamus & 4.01 \\
& Epiphysis & 4.33 \\
& Hypophysis & 4.26 \\
& Thyroid gland & 3.91 \\
& Pancreas & 4.18 \\
Urino-genital system & Adrenals & 5.24 \\
& & 5.24 \\
Immune System & Urino-genital system & 5.18 \\
\hline
\end{tabular}


This study was approved by the Institutional Ethical Committee of SVYASA. The image obtained from each of the fingers is divided into sectors. Each of the sectors corresponds to an organ or an organ system. The data corresponding to the Ring finger has the organs and Organ system related to diabetes.

\section{Analysis}

Three categories of data were analyzed. Right and Left Ring Finger data- Table 1, Chakra data- Table 2, Organs and Organ system data - Table 3

The data in Table 1 has the coronal discharges corresponding to meridians of the ring fingers of both the hands. The meridians correspond to Hypothesis, Thyroid gland, Pancreas, Adrenal, Urinogenital system, Spleen, Nervous system and Hypothalamus.

The data in Table 2 corresponds to the energy centers called chakras. There are seven chakras and the value shown in the table corresponds to their sizes. The table shows the location of the chakras and their values for each subject.

The data in Table 3 has the energy numbers corresponding to the various organs and organ systems. Only a subset of the organs used in the study is shown in the Table3. The energy data corresponding to the Head, Cardiovascular system, Endocrine system, Urino genital system, and the Immune system.

A number corresponding to the photo electronic emissions from each sector of each finger is computed and given in the form of a spread sheet by the Electro Photonic Imaging (EPI) software for all the ten fingers. The EPI instrument captures the data corresponding to all the acupuncture points in each of the fingers. EPI data corresponding to the meridians of the ring finger, Chakras, organs and organ systems, related to diabetes were analyzed using general linear model in IBM SPSS. A built in neural network classifier from IBM SPSS was used to classify diabetic subjects from non-diabetic subjects. Multivariate tests were done to find out the statistical significance of each of the variables. Receiver operating characteristics (ROC) was also plotted for each of the variables and for the output of the neural network.

The "save predicted values for dependent variable" feature was selected to test the accuracy of neural network predictions for multiple runs. The IBM SPSS neural network uses $70 \%$ of the samples randomly, from the given data for training. It uses the remaining 30\% of the data for testing the trained network. The classification report partitions the training and hold out records and gives the details of the accuracy of training for each of the dependent variables.

The tool also dumps out the neural network architecture and the ROC (Receiver Operating Characteristics) along with the area values for the ROC. ROC is the plot of sensitivity Vs Specificity of the dependent variable obtained as a weighted sum of all the inputs of the neural network. This ROC is different from the ROC of the individual parameters. This ROC provides a qualitative assessment of the accuracy of training and classification of the neural network. A good ROC characteristic with an area under its curve close to unity would produce the best classification.

This experiment was repeated with another machine learning tool called Weka and the results compared well with the IBM SPSS tool. The Weka tool had the advantage of defining the training set and testing set rather than allowing the tool to choose its data for training and classification. There is also a scope of integrating this tool with the EPI software without additional data processing for disease diagnosis.

\section{Results}

In all we have the chakra data for 200 subjects. Table 4 has the statistical details like the mean and standard deviation for each of the chakras. It is to be noted that the mean values for all the diabetic subjects is higher than that of the Non-diabetic subjects for all the chakras showing a possible trend to classify the diabetic population from the Non-diabetic. The $\mathrm{P}$ value, truncated to 3 decimal points is shown in the Table 4.

The mean and standard deviations of each of these variables is shown in Table 5. The mean values of each of the variables are listed for Diabetic and Non-diabetic subjects as a comparative table for 115 diabetic subjects and 84 Non-diabetic subjects. It is to be noted that the mean values of all the variables for Diabetic subjects is higher than the mean value of the Non-diabetic subjects as seen from the meridians in the ring finger.

The results of descriptive statistics for the third category diabetic zones are shown in Table 6. The variables eyes, cerebral zone, cardiovascular system, coronary vessels, pancreas, Liver, Urinogenital system, kidneys and immune system all have their mean values for Non-Diabetic subjects greater than that of Diabetic subjects for all the subjects. This data also has a clear trend making it possible to classify diabetic from non-diabetic population.

The ROC characteristics of one of the variables Muladhara chakra is shown in Figure 1. The ROC characteristics of all the variables showed a similar trend. This indicates that though there is a clear difference in the mean values none of these values are significant enough to be able to classify the diabetic population from the Nondiabetic population. This suggested us to use the neural network approach to classify the diabetic and non-diabetic subjects.

The data in Table 7 is the result of neural network classification. The architecture of the neural network was Multi-Layer Perceptron (MLP). The number of Hidden layers was 1 and the number of units in the hidden layer was 5 . The activation function was hyperbolic tangent. The neural network classification was on chakra data with variables shown in Table4. The training samples were $70 \%$ and hold out samples was $30 \%$ of the total samples. Receiver Operating Characteristic (ROC) of the output of the neural network is shown in Figure 2. This characteristic is indicative of the accuracy of the training and classification of the neural network. D and ND in the figure correspond to diabetic and Non-Diabetic samples. The Area under the curve for the ROC was one.

\section{Discussion}

The electron capture from each of the fingers has a definite relationship with the health of the organ/organ system. This electron captured from the finger has to come from the lowest resistance meridian which would be the corresponding organ. The analysis showed significance related to the variables in the endocrine system which has a logical correspondence to diabetes. It also showed correlation with the fourth finger on the right and left hand as pointed out in the previous work. ${ }^{23}$

The results corresponding to the size of the chakras does not give any meaningful insight into the malfunctioning of any single chakra or a group of chakras, it has significant effect on all the chakras. The approach of classifying the data into three different categories makes the study more robust from the point of view of classifying the diabetic and non-diabetic population. 
Table 4 Mean and Standard Deviation for the chakras for Diabetic and Non-Diabetic subjects

\begin{tabular}{|c|c|c|c|c|}
\hline $\begin{array}{l}D=\text { Diabetic, } N D=\text { Non-diabetic } \\
\text { Sample size: } D=\mid \text { I 5,ND=84 }\end{array}$ & & Mean & Std. Deviation & P Value /absolute value \\
\hline Muladhara & $\begin{array}{l}\mathrm{D} \\
\mathrm{ND}\end{array}$ & $\begin{array}{l}5.6397 \\
3.2769\end{array}$ & $\begin{array}{l}.86638 \\
2.76356\end{array}$ & .001 \\
\hline Svadhisthana & $\begin{array}{l}\mathrm{D} \\
\mathrm{ND}\end{array}$ & $\begin{array}{l}5.1361 \\
2.9621\end{array}$ & $\begin{array}{l}.80533 \\
2.56856\end{array}$ & .001 \\
\hline Manipura & $\begin{array}{l}\mathrm{D} \\
\mathrm{ND}\end{array}$ & $\begin{array}{l}5.3900 \\
3.1042\end{array}$ & $\begin{array}{l}.84413 \\
2.66218\end{array}$ & .001 \\
\hline Anahata & $\begin{array}{l}\mathrm{D} \\
\mathrm{ND}\end{array}$ & $\begin{array}{l}5.4257 \\
3.1337\end{array}$ & $\begin{array}{l}.87118 \\
2.72894\end{array}$ & .001 \\
\hline Vishuddha & $\begin{array}{l}\mathrm{D} \\
\mathrm{ND}\end{array}$ & $\begin{array}{l}5.4495 \\
3.2005\end{array}$ & $\begin{array}{l}1.08154 \\
2.55053\end{array}$ & .001 \\
\hline Ajna & $\begin{array}{l}\mathrm{D} \\
\mathrm{ND}\end{array}$ & $\begin{array}{l}4.2300 \\
2.5427\end{array}$ & $\begin{array}{l}.81385 \\
2.11884\end{array}$ & .001 \\
\hline Sahasrara & $\begin{array}{l}\mathrm{D} \\
\mathrm{ND}\end{array}$ & $\begin{array}{l}4.6488 \\
2.6819\end{array}$ & $\begin{array}{l}.77604 \\
2.30118\end{array}$ & .001 \\
\hline
\end{tabular}

Table 5 Mean and Standard deviation for the Organs Corresponding to the Meridians in the Ring Finger

\begin{tabular}{|c|c|c|c|c|}
\hline $\begin{array}{l}\text { Case }=0 \text { Non-diabe } \\
\text { Case }=1.0 \text { Diabetic( }\end{array}$ & & Mean & Std. deviation & $\begin{array}{l}\text { P value- absolute } \\
\text { truncated }\end{array}$ \\
\hline \multirow{2}{*}{ Hypothalamus } & $.00=D$ & 4.9760 & .75926 & 0.0177363954388224 \\
\hline & $1.00=\mathrm{ND}$ & 4.6150 & .86120 & 0.017 \\
\hline \multirow{2}{*}{ Thyroid gland } & $.00=\mathrm{D}$ & 4.8626 & .99482 & $0.007|265957| 5204||$ \\
\hline & $1.00=\mathrm{ND}$ & 4.4211 & .86043 & 0.007 \\
\hline \multirow{2}{*}{ Pancreas } & $.00=D$ & 5.0240 & I.02754 & 0.0865236396429493 \\
\hline & $1.00=N D$ & 4.7383 & .87618 & 0.086 \\
\hline \multirow{2}{*}{ Adrenals } & $.00=D$ & 5.5567 & I.37960 & 0.27627347543829 \\
\hline & $1.00=N D$ & 5.3232 & I.10685 & 0.276 \\
\hline \multirow{2}{*}{ Urino-genital system } & $.00=D$ & 6.2288 & I.54774 & 0.156899546649417 \\
\hline & $1.00=N D$ & 5.8799 & 1.28642 & 0.156 \\
\hline
\end{tabular}

Table 6 Mean and Standard error for the organs effected by Diabetes

\begin{tabular}{|c|c|c|c|c|}
\hline $\begin{array}{l}\text { Dependent Variable } D=\text { Diabetes } \\
\text { ND =Non-diabetes }\end{array}$ & & Mean & Std Error & $\begin{array}{l}\text { P Value Truncated } \\
\text { Absolute }\end{array}$ \\
\hline \multirow{2}{*}{ Eyes } & $\mathrm{D}$ & 4.136 & .076 & \multirow{2}{*}{0.019} \\
\hline & ND & 4.483 & .125 & \\
\hline \multirow{2}{*}{ Cerebral zone (cortex) } & $\mathrm{D}$ & 4.301 & .087 & \multirow{2}{*}{0.031} \\
\hline & ND & 4.665 & .143 & \\
\hline \multirow{2}{*}{ Cardiovascular system } & $\mathrm{D}$ & 4.701 & .070 & \multirow{2}{*}{0.032} \\
\hline & ND & 4.991 & .115 & \\
\hline \multirow{2}{*}{ Coronary vessels } & $\mathrm{D}$ & 4.714 & .085 & \multirow{2}{*}{0.182} \\
\hline & ND & 4.934 & .140 & \\
\hline \multirow{2}{*}{ Pancreas } & $\mathrm{D}$ & 4.738 & .086 & \multirow{2}{*}{0.086} \\
\hline & ND & 5.024 & .142 & \\
\hline \multirow{2}{*}{ Liver } & $\mathrm{D}$ & 6.588 & .136 & \multirow{2}{*}{0.327} \\
\hline & ND & 6.846 & .225 & \\
\hline \multirow{2}{*}{ Urino-genital system } & $\mathrm{D}$ & 5.880 & .127 & \multirow{2}{*}{0.156} \\
\hline & ND & 6.229 & .210 & \\
\hline \multirow{2}{*}{ Kidneys } & $\mathrm{D}$ & 5.672 & .094 & \multirow{2}{*}{0.041} \\
\hline & ND & 6.045 & .155 & \\
\hline \multirow{2}{*}{ Immune system } & $\mathrm{D}$ & 4.052 & .073 & \multirow{2}{*}{0.212} \\
\hline & ND & 4.228 & .121 & \\
\hline
\end{tabular}

Table 7 Neural Network Classification with Multi-Layer Perceptron with one hidden layer and 5 units

\begin{tabular}{|c|c|c|c|c|}
\hline \multicolumn{2}{|c|}{$\begin{array}{l}\text { Sample } \\
\text { D=Diabetic } \\
\text { ND=Non-Diabetic }\end{array}$} & $\begin{array}{l}\text { Predic } \\
\text { D }\end{array}$ & ND & Percent Correct \\
\hline \multirow{3}{*}{ Training } & $\mathrm{D}$ & 81 & 0 & $100.0 \%$ \\
\hline & ND & 0 & 38 & $100.0 \%$ \\
\hline & Overall Percent & $68.1 \%$ & $31.9 \%$ & $100.0 \%$ \\
\hline \multirow{3}{*}{ Holdout } & $\mathrm{D}$ & 29 & 4 & $87.9 \%$ \\
\hline & ND & 2 & 16 & $88.9 \%$ \\
\hline & Overall Percent & $60.8 \%$ & $39.2 \%$ & $88.2 \%$ \\
\hline
\end{tabular}




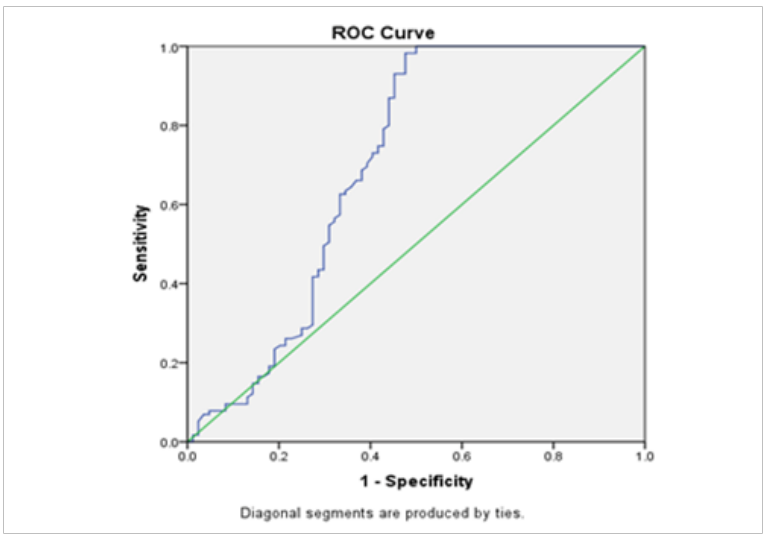

Figure I Receiver Operating Characteristic for the variable Muladhara of Table 2. This indicates that the sensitivity of the dependent variable is not very reliable.

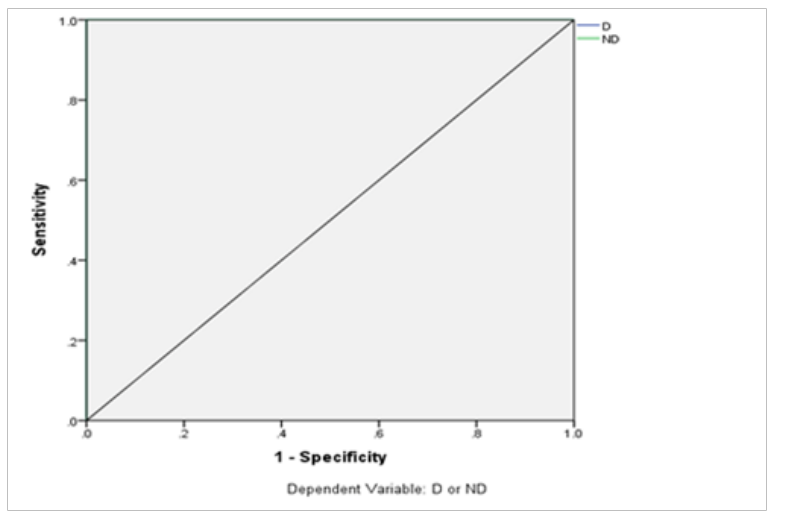

Figure 2 ROC for Table 6- Green line ND is along the Sensitivity axis D is the blue line on the top along the Specificity axis.

It was also observed that the data captured from each finger corresponds to the same variable but the values are significantly different as each of these correspond to different hemispheres in the head region, left hand data corresponds to the right hemisphere and right hand data corresponds to the left hemisphere. The neural network feature of the IBM SPSS tool was used for predicting a given subject as diabetic or non-diabetic by repeated runs of the neural network and the predicted values were saved in the input file.

This experiment could be repeated with larger sample sizes for heterogeneous population for making EPI based instrument, a measurement tool for diabetes. Here the data was analyzed mainly to classify the two populations diabetic and non-diabetic, there was no analysis on correlation of EPI data with the blood sugar measurements as both these approaches are entirely different.

A clear difference in the means and standard deviations of the variables not only makes the two populations separable but also correlates with the likelihood of these values being different as per the diabetic metabolism. As an example pancreas, cardiovascular tissues and hypothalamus should have different values for diabetic and nondiabetic.

The training of the neural network could be a challenge if a subject has multiple comorbidities along with each of the disorders. The data used in this study was not verified to check if the subjects have symptoms related to other disorders in addition to diabetes.

\section{Conclusion}

The diabetic condition has a definite impact on the EPI parameters. The impacted parameters have a logical link with corresponding organs and organ systems. The meridian theory and chakra theory appear to have a scientific significance. Finally this experiment adds value in terms of its ability to relate bio-fields with the disease conditions. it provides a frame work for disease diagnosis using the EPI technique.

\section{Acknowledgements}

All the facilities and support provided by Srinidhi Diagnostic Centre, Bangalore are gratefully acknowledged. Mytra Varun V who meticulously collected the data is here with acknowledged.

\section{Conflicts of interest}

Author declares there are no conflicts of interest.

\section{Funding}

None.

\section{References}

1. Buse JB, Caprio S, Cefalu WT, et al. How Do We Define Cure of Diabetes ? Diabetes Care. 2019;32(11):10-12.

2. American Diabetes Association. Diagnosis and classification of diabetes mellitus. Diabetes Care. 2011;34(Suppl 1):S62-S69.

3. Mezuk B, Eaton WW, Albrecht S, et al. Depression and type 2 diabetes over the lifespan: A meta-analysis. Diabetes Care. 2008; 31(12):23832390 .

4. Pradhan M, Sahu RK. Predict the onset of diabetes disease using Artificial Neural Network (ANN). International Journal of Computer Science \& Emerging Technologies. 2011;2(2):303-311.

5. Woods SC, Morton GJ, Myers MG, et al. Cooperation between brain and islet in glucose homeostasis and diabetes. Nature. 2013;503(7474):5966.

6. Rubik B, Brooks AJ. Digital High-Voltage Electrophotographic Measures of the Fingertips of Subjects Pre- and Post-Qigong. Evid Based Integr Med. 2005;2(4):245-252.

7. Korotkov KG, Matravers P, Orlov D V, et al. Application of electrophoton capture (EPC) analysis based on gas discharge visualization (GDV) technique in medicine: a systematic review. J Altern Complement Med. 2010;16(1):13-25..

8. Korotkov KG. Energy Fields Electrophotonic Analysis in Humans and Nature. Saint-Petersburg. 2011.

9. Alexandrova R, Fedoseev G, Korotkov K. Analysis of the Bioelectrograms of Bronchial Asthma Patients. Cite Web. 2004;75-82.

10. Anufrieva E, Anufriev V, Starchenko M, et al. Thought's Registration by means of Gas-Discharge Visualization. 2014;1-5.

11. Vadis LQ. Lancing: quo vadis? J Diabetes Sci Technol. 2011;5(4):966981

12. Aleksandrova E . GDV Analysis of Arterial Hypertension. Bio-WellEu. 2009;1-9.

13. Deshpande PB, Madappa PK, Korotkov K. Can the Excellence of the Internal Be Measured? - A Preliminary Study. Journal of Consciousness Exploration \& Research. 2013;4(9):977-987.

14. Kostyuk N, Rajnarayanan R V, Isokpehi RD CH. Autism from a biometric perspective. Int J Env Res Public Heal. 2010;7(5):1984-1995. 
15. Priya BS .Kirlian Images based Early Detection of Diabetics using Neural Network. IEEE. 2013; 147-150.

16. Hong W, Chen X, Jin S, et al. Use of an artificial neural network to predict persistent organ failure in patients with acute pancreatitis. Clinics. 2013;68(1):27-31.

17. Parekh R, Yang J, Honavar V. Constructive neural-network learning algorithms for pattern classification. IEEE Trans Neural Networks . 2000;11(2):436-451.

18. Hansen LK, Salamon P. Neural Network Ensembles. IEEE Trans Pattern Anal Mach Intell. 1990;12(10):993-1001.
19. Gardner M, Dorling S. Artificial neural networks (the multilayer perceptron)-a review of applications in the atmospheric sciences. Atmos Environ . 1998;32(14-15):2627-2636.

20. Buhmann MD. Radial basis functions. Acta Numer. 2000 9:1-38.

21. Dey R, Bajpai V. Application of Artificial Neural Network (ANN) technique for Diagnosing Diabetes Mellitus. IEEE. 2008;(155):8-11.

22. http://www.bio-well.com/assets/files/book_biowell.pdf

23. Shanmuga Priya B, Rajesh R. Kirlian images based early detection of diabetics using neural network. 2013 1st International Conference on Orange Technologies (ICOT). IEEE. 2013;147-150. 